\title{
Neutrino Riddles
}

\author{
V. P. Efrosinin \\ Institute for Nuclear Research, Russian Academy of Sciences, Moscow, Russia \\ Email: efrosini@inr.ru
}

Received July 15, 2012; revised August 24, 2012; accepted September 2, 2012

\begin{abstract}
Keywords: Neurino; Oscillations; Masses

Earlier we with use of experimental data on neutrino oscillation [1] had been calculated neutrino masses [2]. Such problem has been solved with use of left-right gauge group $S U_{2}^{L} \times S U_{2}^{R} \times U_{1}$ following the approach of [3] with some changes.

Parametres of neutrino oscillation including experiments with solar and atmospheric neutrinos, in reactor experiments and accelerator experiments are presented in [1] in kind:

$$
\begin{aligned}
& \Delta m_{12}^{2}=7.6(7.3-8.1)\left[10^{-5} \mathrm{eV}^{2}\right], \\
& \Delta m_{23}^{2}=2.4(2.1-2.7)\left[10^{-3} \mathrm{eV}^{2}\right], \\
& \left(\sin \theta_{12}\right)^{2}=0.32(0.28-0.37), \\
& \left(\sin \theta_{23}\right)^{2}=0.50(0.38-0.63), \\
& \left(\sin \theta_{13}\right)^{2}=0.007(\leq 0.033) .
\end{aligned}
$$
\end{abstract}

The possible reasons of features of the nature of neutrino are discussed.

With use of experimental data (1) in [2] following results for masses of neutrino have been received:

$$
\begin{aligned}
& m_{1}=0.0069 \div 0.0073 \mathrm{eV}, \\
& m_{2}=0.0110 \div 0.0116 \mathrm{eV}, \\
& m_{3}=0.0471 \div 0.0532 \mathrm{eV} .
\end{aligned}
$$

Phenomenon of small masses of neutrinos appears rather essential. There comes a threshold of stability of discrete character of masses of neutrinos and enough free transition from one generation to another. For comparison we result masses of partners of neutrino on the weak charged currents at which birth oscillations is absent:

$$
\begin{aligned}
& m_{e}=0.511 \mathrm{MeV}, \\
& m_{\mu}=105.7 \mathrm{MeV}, \\
& m_{\tau}=1777 \mathrm{MeV} .
\end{aligned}
$$

Also from (1) and (2) noticed that

$$
\begin{aligned}
& \frac{\left|\sin \theta_{13}\right|}{\left|\sin \theta_{12}\right|} \simeq \sqrt{\frac{0.007}{0.32}}=0.15, \\
& \frac{m_{1}}{m_{3}} \simeq \frac{0.007}{0.05}=0.14 .
\end{aligned}
$$

That is certain proportionality of masses of generations of neutrinos and sine of corners of mixing is observed. The more masses of pair neutrinos differ from each other the less between them a mixing sine of the angle. For example, mixing usual neutrinos and sterile neutrinos with rather big masses will be suppressed.

It is necessary to tell that in direct measurement neutrino oscillation there is a problem of statistical uncertainty at definition of parametres. Use of two various mixing matrixes in calculation of parametres will appear useful. Also there will be useful in processing of results of these experiments use of additional criteria except $\chi^{2}$ minimum method.

There is some affinity of neutrino with photons including in the cosmology plan. Photon is some twirled whirlwind of energy with spin $S=1$ and with zero mass. Neutrino has spin $S=1 / 2$ and very small mass (2). Relic streams of neutrinos in a sense are satellite in relation to relic radiation of photons. Direct detecting of relic neutrinos is unsoluble at present a problem.

Since introduction of technics of research of neutrino oscillation by B. Pontecorvo a little that has changed in understanding of the essence of this phenomenon. The version of this understanding offered by us is some step to this direction.

From the point of view of the justification of our approach on experiment the result of measurement of mass of smallest of leptons - an electron testifies at least:

$m_{e}=0.510998910 \pm 0.000000013 \mathrm{MeV}$. The measurement error is equal to $0.013 \mathrm{eV}$ and means in the order of size difference of mass of one electron from another, accuracy of the nature for elementary particles of small 
masses. In the order of size it is mass of neutrino.

Obviously, that masses of sterile neutrinos do not get to area of masses of three generations of neutrinos. If sterile neutrino exist, their masses of essentially more masses of usual neutrinos. The prediction in our work is instructive that their mixing with usual neutrinos will be suppressed. This remark will not appear superfluous for researchers-experimenters.

\section{REFERENCES}

[1] T. Schwetz, hep-ph/0710.5027, 2007.

[2] V. P. Efrosinin, Yadernaya Fizika, Vol. 73, 2010, pp. 975-977.

[3] H. Fritzsch, Physics Letters, Vol. 73B, 1978, p. 317. 